\title{
Scleroderma-polymyositis overlap syndrome versus idiopathic polymyositis and systemic sclerosis: a descriptive study on clinical features and myopathology
}

Kavish J Bhansing ${ }^{1 *}$, Martin Lammens ${ }^{2}$, Hanneke KA Knaapen ${ }^{1}$, Piet LCM van Riel ${ }^{3}$, Baziel GM van Engelen ${ }^{4}$ and Madelon C Vonk ${ }^{1}$

\begin{abstract}
Introduction: The objective was to characterize the clinical and myopathologic features of patients with scleroderma-polymyositis (SSC-PM) overlap compared with a population of patients with systemic sclerosis (SSc) and polymyositis (PM).

Methods: A three-way comparison of patients with SSc-PM overlap $(n=25)$ with patients with SSC $(n=397)$ and PM $(n=40)$ on clinical and myopathologic features and causes of death. One neuropathologist blinded for the diagnosis evaluated all recent available muscle biopsies. Biopsies were scored for presence of inflammation, necrotic muscle fibers, rimmed vacuoles, fibrosis, and immunohistochemical staining. Clinical or myopathologic characteristics were compared by using the $x^{2}$ test or one-way analysis of variance (ANOVA).

Results: The prevalence of SSc-PM overlap in the Nijmegen Systemic Sclerosis cohort was 5.9\%. The mortality was $32 \%$ (eight of 25) in SSc-PM, of which half was related to cardiac diseases. The prevalence of pulmonary fibrosis was significantly increased in SSc-PM (83\%) $(P=0.04)$ compared with SSC (49\%) and PM (53\%). SSc or myositis-specific antibodies were nearly absent in the SSc-PM group. In almost all biopsies (96\%) of SSc-PM patients, necrotic muscle fibers were present, which was significantly increased compared with PM patients $(P=0.02)$.

Conclusions: Patients with SSC-PM have increased prevalence of pulmonary fibrosis and cardiac disease as the cause of death compared with patients with SSC and PM . In addition, we found that necrotizing muscle fibers with inflammation characterize SSc-PM overlap in muscle biopsies. Further research should focus on underlying mechanisms causing necrosis, inflammation, and fibrosis and their relation to pulmonary involvement and mortality in patients with SSc-PM overlap.
\end{abstract}

\section{Introduction}

Systemic sclerosis (SSc) is a systemic autoimmune disease characterized by vascular lesions and fibrosis of multiple organs, predominantly in skin, lungs, heart, intestinal tract, joints, and muscles [1,2]. The prevalence of myopathies in SSc patients varies from $5 \%$ to $81 \%$, depending on the use of different definitions of muscle involvement [3-11]. Myositis in SSc patients resembles clinical and biologic features

\footnotetext{
* Correspondence: k.bhansing@reuma.umcn.nl

'Department of Rheumatic Diseases, Radboud Medical Center, Nijmegen, The Netherlands

Full list of author information is available at the end of the article
}

of patients with polymyositis (PM), hence the term scleroderma/polymyositis overlap (SSc-PM). Previous studies demonstrated a worse survival and increased prevalence of myocardial involvement in SSc-PM overlap compared with SSc $[9,12]$. Therefore, it seems clinically relevant to identify SSc-PM overlap for close monitoring and early treatment. To date, no studies have been conducted to characterize the full spectrum of SSc-PM overlap by means of a threeway comparison with SSc and idiopathic PM. Better understanding of similarities and differences in clinical and biologic features as well as outcome may lead to improved treatment and prognosis for SSc-PM overlap patients. 
The aim of this study is to characterize the clinical and myopathologic features of patients with SSc-PM overlap patients compared with a population of patients with SSc and PM.

\section{Methods \\ Design}

The Nijmegen Systemic Sclerosis cohort is an ongoing, prospective inception cohort started in 1989 at the Department of Rheumatic Diseases at the Radboud University Medical Center. The data collection contains information of symptoms, physical examination, laboratory workup, as well as annually performed pulmonary-function test results, echocardiography, right-heart catheterization, and high-resolution computed tomography (HRCT) scans at baseline and when indicated.

The Nijmegen Myositis cohort is also a prospectively followed-up cohort of patients with inflammatory myopathies. This cohort includes all patients with inflammatory myopathies from the Computer Registry of All Myopathies and Polyneuropathies (CRAMP) treated at the Radboud University Medical Center [13]. The CRAMP is a Dutch multicenter neuromuscular registry and was developed in 2004. The data available of the Nijmegen Myositis cohort consist of demographic and clinical features at diagnosis combined with follow-up information on treatment, biochemical markers, pulmonary-function test results, and, if indicated, HRCT scans and echocardiography.

\section{Participants}

All patients with SSc can be classified by the ACR preliminary classification criteria for SSc or Leroy criteria for early SSc. The patients with PM all fulfilled the Bohan and Peter diagnostic criteria, whereas the SSc-PM overlap participants fulfilled both criteria [14-17].

All SSc-PM patients of the Nijmegen Systemic Sclerosis cohort were included. SSc patients with serum CK more than 2 times upper limit, myalgia, and proximal muscle weakness were analyzed for the presence of polymyositis with electromyography (EMG) and muscle biopsy. All consecutive PM patients of the Nijmegen Myositis cohort were included. The study was exempted from approval of the local Medical Ethics Committee Arnhem-Nijmegen in the Netherlands, because this was an observational, noninterventional study. Therefore, no informed consent was required for this study.

\section{Myopathologic analysis}

The slides of all available muscle biopsies of SSc-PM ( $n=$ $24)$ and most-recent consecutive patients with PM $(n=24)$ were used to pair with a 1:1 ratio. One neuropathologist $(\mathrm{ML})$, blinded for diagnosis, evaluated all muscle biopsies. Biopsies were scored for the presence of inflammatory infiltrates, necrotic muscle fibers, fibrosis, rimmed vacuoles, and, if performed for enzyme histochemistry, for cytochrome C-oxidase (COX) and succinate dehydrogenase $(\mathrm{SDH})$ and immunohistochemistry for MHC class I, membrane attack complex (MAC), CD 4, CD 8, CD 20, and $\mathrm{CD}$ 68. Inflammatory infiltrates were defined as the presence of mononuclear cell infiltrates surrounding or invading muscle fibers. Necrosis in muscle biopsy was defined as presence of acute necrotic muscle fibers, identified on hematoxylin-phloxine staining (paling of the cytoplasm and absence of basophilia), myophagia, or the presence of regenerating basophilic fibers. Fibrosis was defined as increase of collagen and fibroblasts in the endomysium. $\mathrm{MHC}$ class I upregulation was regarded as positive if at least the sarcolemma expressed MHC class I antigen [18]. MAC upregulation was regarded as present if MAC was expressed in the endothelium of capillaries or in the sarcoplasm of muscle fibers $[19,20]$.

\section{Statistical analysis}

Statistical analysis was performed with SPSS version 20 for Windows (SPSS Inc., Chicago, IL, USA). Study population and muscle-biopsy characteristics were compared with the $X^{2}$ test or Fisher Exact test for nominal data and the Mann-Whitney $U$ test for numeric data or one-way ANOVA. Statistical significance was defined as $P \leq 0.05$.

\section{Results}

The mean age at diagnosis was 53 years (SD, 12.3) (SScPM overlap), 50 years (SD, 13.2) (SSc), and 51 years (SD, 16.5) (PM). The median disease duration was 5.0 years (IQR, 2.8 to 12.4 ), 7 years (IQR, 3 to 14 ), and 3.5 years (IQR, 2 to 11), respectively. The SSc-PM group was characterized by an almost 1:1 female/male ratio, whereas the SSc group by 2:1 female-to-male ratio, and the PM group by an almost opposite ratio of 1:2 (Table 1). The prevalence of SSc-PM overlap in the Nijmegen Systemic Sclerosis cohort was $5.9 \%$.

\section{Serology}

SSc-specific autoantibodies (anticentromere and antitopoisomerase 1), were nearly absent in the SSc-PM group and present in $19 \%$ and $22 \%$ of the SSc patients. None of the PM patients tested positive for anticentromere and antitopoisomerase 1, except one PM patient. Myositis-associated antibody, anti-SSA and myositis-specific antibody, antiJo1, were significantly less present in the SSc-PM group compared with the PM groups (Table 1).

\section{Scleroderma and myositis-specific features}

Raynaud phenomenon was present in all groups: 21 patients (84\%) in SSc-PM overlap, 379 patients (96\%) in SSc, and 13 patients (33\%) in PM. The 13 (33\%) patients with Raynaud phenomena in the PM group also had features such as mechanic hands, arthritis, ILD, and were 
Table 1 Study population characteristics

\begin{tabular}{|c|c|c|c|c|c|c|c|}
\hline Characteristics & Number & SSc-overlap $(n=25)$ & Number & SSc $(n=397)$ & Number & PM $(n=40)$ & $P$ value \\
\hline Age in years, mean (SD) & 25 & $53(12.3)$ & 397 & $50(13.2)$ & 40 & $51(16.5)$ & 0.013 \\
\hline Gender (male/female) & 25 & $12 / 13$ & 397 & $131 / 266$ & 40 & $25 / 15$ & 0.003 \\
\hline \multicolumn{8}{|l|}{ Type diagnosis } \\
\hline Limited cutaneous & & $19(76 \%)$ & & $276(73 \%)$ & & NA & NS \\
\hline Diffuse cutaneous & & $6(24 \%)$ & & $109(27 \%)$ & & & \\
\hline Disease duration, years median (IQR) & 25 & $5(3-12)$ & 397 & $7(3-14)$ & 40 & $3.5(2-11)$ & 0.001 \\
\hline Mortality & 25 & $8(32 \%)$ & 397 & $65(16 \%)$ & 40 & $7(18 \%)$ & NS \\
\hline Survival in years, median (IQR) & 8 & $2.5(1.3-9.6)$ & 65 & $7(3-13.5)$ & 7 & $0.3(0-12)$ & NS \\
\hline \multicolumn{8}{|l|}{ Serology } \\
\hline ANA & 25 & $25(100 \%)$ & 397 & $356(90 \%)$ & 34 & $21(62 \%)$ & 0.006 \\
\hline Anti-topoisomerase & 21 & 0 & 397 & $87(22 \%)$ & 31 & $1(3 \%)$ & 0.048 \\
\hline Anti-centromere & 19 & $2(10 \%)$ & 397 & 76 (19\%) & 31 & 0 & NS \\
\hline Anti-SSA & 25 & $2(8 \%)$ & 397 & $15(4 \%)$ & 31 & $11(36 \%)$ & $<0.001$ \\
\hline Anti-SSB & 25 & 0 & & NA & 31 & $1(3 \%)$ & NS \\
\hline Anti-RNP & 25 & $2(8 \%)$ & 397 & $27(7 \%)$ & 31 & $2(6 \%)$ & NS \\
\hline Anti-SM & 25 & 0 & & NA & 31 & $2(6 \%)$ & NS \\
\hline Anti-Jo1 & 25 & $2(8 \%)$ & & NA & 31 & $13(42 \%)$ & 0.013 \\
\hline
\end{tabular}

NA, not applicable; NS, not significant.

Table 2 Disease-specific characteristics

\begin{tabular}{|c|c|c|c|c|c|c|c|}
\hline Characteristics & Number & SSc-overlap $(n=25)$ & Number & SSc $(n=397)$ & Number & PM $(n=40)$ & $P$ value \\
\hline \multicolumn{8}{|l|}{ Scleroderma features } \\
\hline Raynaud phenomena & 25 & $21(84 \%)$ & 397 & $379(96 \%)$ & 40 & $13(33 \%)$ & $<0.001$ \\
\hline Digital ulcers & 25 & $6(24 \%)$ & 397 & $164(41 \%)$ & 40 & $3(8 \%)$ & $<0.001$ \\
\hline Pitting scars & & - & 397 & $160(40 \%)$ & & NA & NA \\
\hline Rodnan skin score (median) & 15 & $6(5-11)$ & 372 & $7(4-12)$ & & NA & NS \\
\hline Renal crisis & 25 & $1(4 \%)$ & 397 & $17(4 \%)$ & & NA & NS \\
\hline \multicolumn{8}{|l|}{ Myositis features } \\
\hline Serum CK elevation ${ }^{\ddagger}$ & 25 & $24(96 \%)$ & 397 & $48(12 \%)$ & 40 & $34(85 \%)$ & $<0.001$ \\
\hline Proximal muscle weakness & 25 & $25(100 \%)$ & 397 & $21(5 \%)$ & 40 & 37 (93\%) & $<0.001$ \\
\hline Myopathic EMG findings & 25 & $22(88 \%)$ & & NA & 29 & $24(83 \%)$ & NS \\
\hline Mechanic hands & & NA & & NA & 40 & $7(18 \%)$ & NS \\
\hline \multicolumn{8}{|l|}{ Internal complications } \\
\hline Arthritis & 25 & $5(20 \%)$ & 397 & $46(12 \%)$ & 40 & $7(18 \%)$ & NS \\
\hline \multicolumn{8}{|l|}{ Interstitial lung disease } \\
\hline HRCT fibrosis & 18 & $15(83 \%)$ & 346 & $170(49 \%)$ & 19 & $10(53 \%)$ & 0.044 \\
\hline $\mathrm{TLC} \leq 70 \%$ of predicted & 21 & $4(19 \%)$ & 364 & $68(19 \%)$ & 23 & $9(39 \%)$ & NS \\
\hline TLCO $\leq 70 \%$ of predicted & 17 & $17(100 \%)$ & 347 & $260(75 \%)$ & 20 & $13(65 \%)$ & 0.001 \\
\hline Diastolic dysfunction by cardiac ultrasound & 19 & $7(37 \%)$ & 352 & $146(42 \%)$ & 19 & $4(21 \%)$ & NS \\
\hline PH suspicion by cardiac ultrasound & 20 & $3(15 \%)$ & 352 & $89(25 \%)$ & 19 & $2(11 \%)$ & 0.042 \\
\hline PH by cardiac catherization & 5 & 0 & 182 & $57(31 \%)$ & 2 & $1(50 \%)$ & 0.002 \\
\hline Myocarditis & 25 & 1 (4\%) & & NA & 40 & 0 & NS \\
\hline Malignancy & 25 & $2(8 \%)$ & & NA & 40 & $5(13 \%)$ & NS \\
\hline
\end{tabular}

NA, not applicable; NS, not significant; TLC, total lung capacity; HRCT, high-resolution CT scan; TLCO, transfer factor of the lung for carbon monoxide; PH, 
anti-Jo1 antibodies positive, reflecting the anti-synthetase syndrome. The median modified Rodnan skincore (mRSS) was 6 (IQR, 5 to 11) in the SSc-PM group and 7 (IQR, 4 to 12 ) in the SSc group (Table 2).

Signs of myositis such as serum CK elevation ( $>2$ times ULN) and proximal muscle weakness were present in 48 SSc patients (12\%) and in 21 SSc patients (5\%), respectively (Table 1). Arthritis was present in all three groups, with a prevalence ranging from $12 \%$ to $20 \%$.

\section{Interstitial lung disease}

Interstitial lung disease (ILD) was diagnosed in patients whether or not moderate to severe pulmonary fibrosis was present on the HRCT scan. The presence of ILD was statistically significantly higher in the SSc-PM overlap group compared with the other two groups. Furthermore, a significantly higher prevalence of decreased lung carbon monoxide diffusion capacity (defined as TLCO $\leq 70 \%$ ) was found in the SSc-PM overlap group (Table 2).

\section{Cardiac complications}

Diastolic dysfunction on echocardiography was present in all three groups. Right heart catheterization (RHC) was performed only when pulmonary hypertension $(\mathrm{PH})$ was suspected at echocardiography or in cases with unexplained dyspnea or an isolated decrease of carbon monoxide $(\mathrm{CO})$ diffusion capacity at pulmonary-function testing. $\mathrm{PH}$ was diagnosed in none of the SSc-PM overlap patients, in 57 SSc patients, and in one PM patient. When no RHC was performed, we assumed that PH was absent. With this assumption, we found a statistically significant increase of $\mathrm{PH}$ in SSc patients compared with the other groups.

\section{Malignancy}

In the SSc-PM overlap group, one patient developed a squamous cell carcinoma in the neck, and one patient developed a basal cell carcinoma of the skin on the forehead. In five patients in the PM group, a malignancy was diagnosed during follow-up, consisting of renal cell carcinoma $(n=1)$, melanoma $(n=1)$, breast cancer $(n=1)$, esophagus carcinoma $(n=1)$, and lung carcinoma $(n=1)$. Data concerning numbers and types of malignancy cases were not available in the SSc group.

\section{Mortality}

Eight patients (32\%) in the SSc-PM overlap group died, which was not statistically significantly increased compared with the other groups (Table 1). The causes of death were progressive heart disease $(n=2)$, myocardial infarction $(n=2)$, respiratory failure due to interstitial lung disease (ILD) $(n=1)$, sepsis $(n=2)$, and unknown cause $(n=1)$. Causes of mortality in the SSc group were progressive heart failure $(n=7) 10.8 \%$, myocardial infarction $(n=2) 3.1 \%$, respiratory failure due to ILD $(n=11) 16.9 \%$, or pulmonary hypertension $(n=13) 20 \%$, malignancies $(n=6) 9.2 \%$, infections $(n=6) 9.2 \%$, and other or unknown causes $(n=20) 30.8 \%$. In the PM group, seven patients died. Their causes of death were progressive heart disease $(n=2)$, aspiration pneumonia $(n=2)$, malignancy $(n=2)$, and unknown cause $(n=1)$.

\section{Myopathology}

All muscle biopsies were assessed by the same neuropathologist who was blinded for the clinical diagnosis, and his findings are shown in Table 3 and Figure 1. We found a statistically significant difference in the presence of necrotic muscle fibers, present in $96 \%$ of the SSc-PM biopsies compared with $67 \%$ of the PM biopsies. This significant difference was caused mostly by differences in the presence of acute necrotic muscle fibers (50\% versus $29.2 \%$ ).

The presence of inflammatory markers such as lymphocytic infiltrates (63\% versus $54 \%)$ and positive MHC I staining (92\% versus $78 \%$ ) were not significantly different among the SSc-PM overlap patients compared with the PM patients. Fibrosis displayed quite similar rates of presence in both groups. None of the muscle biopsies revealed rimmed vacuoles, ruling out inclusion-body myositis [21] On characterization of different types of lymphocytes, only limited data were available. Deposition of MAC was found only in necrotic muscle fibers and not in the capillaries. $\mathrm{COX}$ and SDH staining was more often applied on the specimens and in a small number of cases $(n=3)$ in SSc$\mathrm{PM}$ and $(n=6)$ in PM revealed the presence of COXnegative muscle fibers.

\section{Discussion}

In this study, we describe and compare both the clinical characteristics and myopathologic findings of patients with SSc-PM overlap, SSc and PM. We found an increased prevalence of pulmonary fibrosis among patients with SScPM overlap, which is in accordance with a Japanese study that also showed an increased presence of ILD among patients with SSc-PM overlap compared with patients with SSc [7]. However, an increased presence of ILD was not confirmed in a French study [22]. ILD can cause early death and can diminish the quality of life because of symptoms of dyspnea and fatigue. However, no significant difference was found in ILD-associated death between the patients with SSc-PM overlap, SSc, and PM. However, our study has only a small study population, which limits a proper survival analysis with ILD. The increased prevalence of ILD in overlap patients should warrant regular screening for this complication and timely initiation of specific immunosuppressive therapy. Further studies should focus on the extent of pulmonary fibrosis and related morbidity among SSc-PM patients. 
Table 3 Myopathologic markers of muscle biopsy slides

\begin{tabular}{|c|c|c|c|c|c|}
\hline Characteristics & Number & SSc-overlap $(n=24)$ & Number & PM $(n=24)$ & Significance ( $P$ value) \\
\hline Necrotic muscle fibers & 24 & $23(96 \%)$ & 24 & $16(67 \%)$ & $0.023^{\mathrm{bt}}$ \\
\hline Lymphocytic infiltrates & 24 & $15(63 \%)$ & 24 & $13(54 \%)$ & NS \\
\hline Positive MHC class I staining & 12 & $11(92 \%)$ & 18 & $12(67 \%)$ & NS \\
\hline Inflammation $^{a}$ & 24 & 19 (79\%) & 24 & $18(75 \%)$ & NS \\
\hline MAC staining & 10 & $5(50 \%)$ & 13 & $5(39 \%)$ & NS \\
\hline Fibrosis & 19 & $5(26 \%)$ & 24 & $2(8 \%)$ & NS \\
\hline Presence of COX-negative fibers & 10 & $3(30 \%)$ & 10 & $6(60 \%)$ & NS \\
\hline
\end{tabular}

MAC, Membrane attack complex; NA, not applicable; NS, not significant.

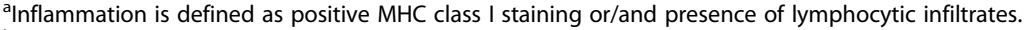

${ }^{b}$ Statistically significant.

Several studies have described increased myocardial involvement, such as diastolic or systolic dysfunction and myocarditis, in patients with SSc-associated myopathies $[7,9,22,23]$. We found no statistically significant difference in myocardial involvement between the three patient cohorts in our study, but we used echocardiography as a screening tool, and cardiac MRI was not performed routinely. Echocardiography lacks specificity to diagnose myocarditis accurately, so the prevalence of this complication could prove to be higher if a cardiovascular MRI were performed as well [24]. In our cohort, we found progressive heart disease to be the cause of death
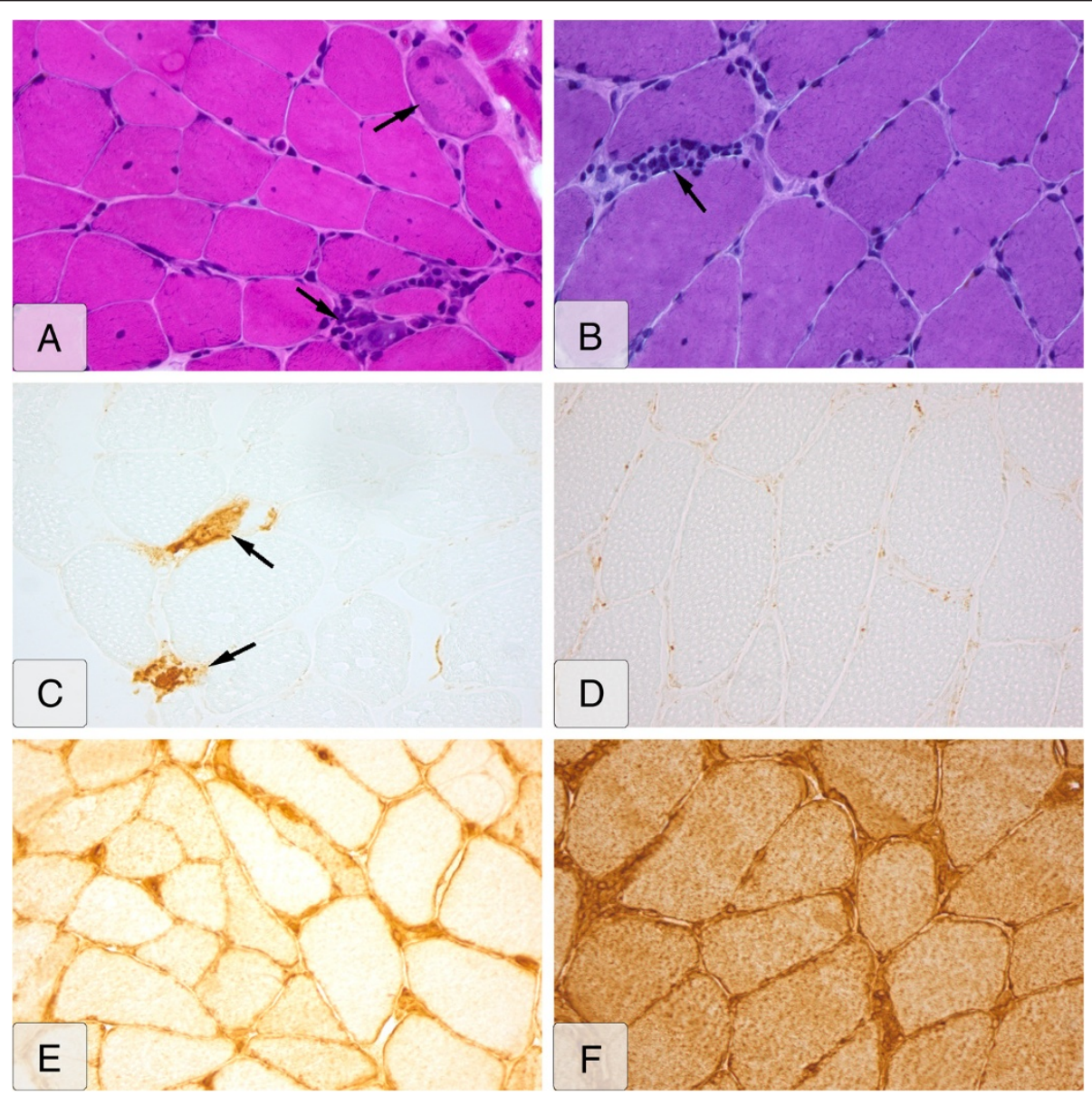

Figure 1 Histopathologic muscle-biopsy images of SSc-PM overlap patient versus PM patient. Left side (A, C, E): SSC-overlap patient. Right side (B, D, F): PM patient. (A) Hematoxylin \& eosin (H\&E) stain; upper marker, necrosis; lower marker, lymphocytic infiltrate; (B) H\&E stain; marker, lymphocytic infiltrate; (C) MAC stain; markers, MAC upregulation; (D) MAC stain, no upregulation; (b v 02D7; (E) MHC class I stain, sarcolemmal MHC class I upregulation; (F) MHC class I stain, sarcolemmal and diffuse cytoplasmic MHC class I upregulation. 
in $25 \%$ of our overlap patients compared with only $10.8 \%$ of SSc patients.

The prevalence of SSc-PM overlap in our Nijmegen Systemic Sclerosis cohort was low (5.9\%) compared with that in older investigations [3-7,9]. In more-recent studies, prevalences of $7.6 \%$ and $7.2 \%[22,25]$ were observed, which is comparable to our finding. This discrepancy in prevalences compared with older studies can be explained by the different criteria used to define SSc myopathy and the different study designs. In our study, we used strict criteria to select only inflammatory myopathies among SSc patients and not associated myopathies. Furthermore, this investigation includes only clinically relevant causes, whereas Medsger et al. [3] reported 90\% prevalence of proximal muscle weakness, of which only $20 \%$ complained of musclerelated symptoms.

Different antibodies are associated with SSc-PM overlap, such as anti-PM/Scl and anti-Ku [26,27]. Morerecent studies show novel antibodies related to SSc-PM, anti-U3 RNP, and anti-RuvBL1/2, respectively $[28,29]$. These antibodies relate to different clinical manifestations of SSc-PM-overlap patients; for example, Caucasian patients with limited cutaneous involvement and pulmonary fibrosis (antiPM/Scl) or African male patients with diffuse cutaneous involvement and pulmonary arterial hypertension (anti-U3 RNP) [26,28]. Unfortunately, no data concerning SSc-PM overlap-associated antibodies were available, which can be regarded as a limitation of our study.

We studied muscle biopsies of both overlap and PM patients, blinded for the diagnosis. For the first time, our study revealed that the myopathology in patients with SScPM overlap syndrome shows specific features compared with PM patients. Necrotizing muscle fibers were observed in almost all SSc-PM overlap samples. In the majority of the SSc-PM muscle biopsies, features of inflammation were also found, enabling us to exclude the histopathologic diagnosis of a necrotizing myopathy, according to the European Neuromuscular Centre workshop 2003 [30]. A French study showed a slightly lower prevalence of 25 (63\%) of 35 necrotic muscle fibers among their cohort of SSc-PM overlap patients [8]. However, when including the presence of regenerating (that is, basophilic) muscle fibers, a feature present after necrosis, the prevalence of necrosis is in accordance with our findings.

MAC staining is often used to analyze muscle biopsies for signs of dermatomyositis (DM) if present as linear deposits at endothelial cells of endomysial capillaries $[19,20]$. This was not present in our study. We observed MAC staining in the sarcoplasm, which is indicative of necrotic fibers. No presence of rimmed vacuoles was found in this study. This verifies that our PM muscle biopsies are homogeneous for that diagnosis and are unlikely to include patients with IBM or DM.
The presence of COX-negative muscle fibers might be indicative of mitochondrial abnormalities. Growing evidence suggests that mitochondrial abnormalities can be found in dermatomyositis, inclusion-body myositis, and very seldom in polymyositis [21,31]. The results of our study revealed a small number of cases with COX-negative fibers. Only two PM patients had more than a single affected muscle fiber, but both patients were older than 65 years, and in these cases, the COX-negative fibers may be considered an effect of ageing. Therefore, we conclude that no evidence exists for mitochondrial pathology in patients with SSc-PM overlap.

This study has several limitations. All the subjects were recruited from a single, tertiary referral center, which may have led to a bias toward more-severe cases. However, this is true for all three patient cohorts, and therefore, it would less likely bias observed differences among the patient cohorts. The small sample size of the SSc-PM overlap cohort makes us cautious about overinterpretation of results. Nonetheless, by using strict criteria, we were able to identify a well-defined homogeneous overlap cohort. Furthermore, the blinded-for-diagnosis expert assessment of overlap muscle biopsies in comparison with PM biopsies is unique and greatly increased the strength of our findings.

\section{Conclusions}

In conclusion, we found a prevalence of $5.9 \%$ of SSc-PM overlap in the Nijmegen Systemic Sclerosis cohort. Second, we observed an increased proportion of pulmonary fibrosis among patients with SSc-PM overlap. Third, we revealed that cardiac complications are a major cause of death in patients with SSc-PM overlap. Consequently, we urge more active and regular cardiopulmonary screening among these SSc-PM patients. Fourth, we found that necrotizing muscle fibers with inflammation characterize SScPM overlap in muscle biopsies. Further research should focus on underlying mechanisms causing necrosis, inflammation, and fibrosis and their relation to pulmonary involvement and mortality in SSc-PM overlap patients to optimize treatment, increase survival, and improve quality of life.

\section{Abbreviations \\ COX: Cytochrome C-oxidase; EMG: electromyography; HRCT scan: high-resolution computed tomography scan; ILD: interstitial lung disease; MAC: membrane attack complex; mRSS: modified Rodnan skin score; PM: polymyositis; SDH: succinate dehydrogenase; SSc: systemic sclerosis; SSc-PM: scleroderma-polymyositis.}

\section{Competing interests}

All authors declare not to have received any financial support or other benefits from commercial sources for work reported here or to have any interests that could create a potential conflict of interest or appearance of conflict of interest with regard to this work.

\section{Authors' contributions}

KB participated in the design of the study and carried out data collection, statistical analysis, and drafting of the manuscript. ML carried out evaluation 
of the muscle biopsies and participated in the design and drafting of the manuscript. HK was involved in the design of the study, and helped in the coordination and drafting of the manuscript. PVR participated in the design of the study, interpretation of the data, drafting, and critical revising of the manuscript. BvE participated in the design of the study, interpretation of the data, and critical revising of the manuscript. MV conceived the study, and participated in the coordination, interpretation of the data, and drafting of the manuscript. All authors read and approved the final manuscript.

\section{Author details}

'Department of Rheumatic Diseases, Radboud Medical Center, Nijmegen, The Netherlands. ${ }^{2}$ Department of Pathology, Antwerp University Hospital, Antwerp, Belgium. ${ }^{3}$ Scientific Institute for Quality of Healthcare, Radboud University Medical Center, Nijmegen, The Netherlands. ${ }^{4}$ Department of Neurology, Radboud University Medical Center, Nijmegen, The Netherlands.

Received: 30 July 2013 Accepted: 24 April 2014

Published: 13 May 2014

\section{References}

1. Furst DE, Clements PJ: Hypothesis for the pathogenesis of systemic sclerosis. J Rheumatol Supp/ 1997, 48:53-57.

2. Medsger TA Jr, Masi AT: Epidemiology of systemic sclerosis (scleroderma). Ann Intern Med 1971, 74:714-721.

3. Medsger TA Jr, Rodnan GP, Moossy J, Vester JW: Skeletal muscle involvement in progressive systemic sclerosis (scleroderma). Arthritis Rheum 1968, 11:554-568.

4. Thompson JM, Bluestone R, Bywaters EG, Dorling J, Johnson M: Skeletal muscle involvement in systemic sclerosis. Ann Rheum Dis 1969, 28:281-288.

5. Tuffanelli DL: Scleroderma and its relationship to the "collagenoses": dermatomyositis, lupus erythematosus, rheumatoid arthritis and Sjogren's syndrome. Am J Med Sci 1962, 243:133-146.

6. Clements PJ, Furst DE, Campion DS, Bohan A, Harris R, Levy J, Paulus HE: Muscle disease in progressive systemic sclerosis: diagnostic and therapeutic considerations. Arthritis Rheum 1978, 21:62-71.

7. Mimura Y, Ihn H, Jinnin M, Asano Y, Yamane K, Tamaki K: Clinical and laboratory features of scleroderma patients developing skeletal myopathy. Clin Rheumatol 2005, 24:99-102.

8. Ranque B, Authier FJ, Le-Guern V, Pagnoux C, Berezne A, Allanore Y, Launay D, Hachulla E, Kahan A, Cabane J, Gherardi R, Guillevin L, Mouthon L: A descriptive and prognostic study of systemic sclerosis-associated myopathies. Ann Rheum Dis 2008, 68:1474-1477.

9. Follansbee WP, Zerbe TR, Medsger TA Jr: Cardiac and skeletal muscle disease in systemic sclerosis (scleroderma): a high risk association. Am Heart J 1993, 125:194-203.

10. Tuffanelli DL, Winkelmann RK: Systemic scleroderma: a clinical study of 727 cases. Arch Dermatol 1961, 84:359-371.

11. Mimori T: Scleroderma-polymyositis overlap syndrome: clinical and serologic aspects. Int J Dermatol 1987, 26:419-425.

12. Hashimoto A, Tejima S, Tono T, Suzuki M, Tanaka S, Matsui T, Tohma S, Endo $\mathrm{H}$, Hirohata S: Predictors of survival and causes of death in Japanese patients with systemic sclerosis. J Rheumatol 2011, 38:1931-1939.

13. van Engelen $B G$, van Veenendaal $H$, van Doorn $P A$, Faber $C G$, van der Hoeven JH, Janssen NG, Notermans NC, van Schaik IN, Visser LH, Verschuuren JJ: The Dutch neuromuscular database CRAMP (Computer Registry of All Myopathies and Polyneuropathies): development and preliminary data. Neuromusc Disord 2007, 17:33-37.

14. Subcommittee for scleroderma criteria of the American Rheumatism Association Diagnostic and Therapeutic Criteria Committee: Preliminary criteria for the classification of systemic sclerosis (scleroderma). Arthritis Rheumatism 1980, 23:581-590.

15. LeRoy EC, Medsger TA Jr: Criteria for the classification of early systemic sclerosis. J Rheumatol 2001, 28:1573-1576.

16. Bohan A, Peter JB: Polymyositis and dermatomyositis (first of two parts). N Engl J Med 1975, 292:344-347.

17. Bohan A, Peter JB: Polymyositis and dermatomyositis (second of two parts). N Engl J Med 1975, 292:403-407.

18. van der Pas J, Hengstman GJ, Ter Laak HJ, Borm GF, van Engelen BG: Diagnostic value of MHC class I staining in idiopathic inflammatory myopathies. J Neurol Neurosurg Psychiatry 2004, 75:136-139.
19. Kissel JT, Mendell JR, Rammohan KW: Microvascular deposition of complement membrane attack complex in dermatomyositis. N Engl J Med 1986, 314:329-334.

20. Jain A, Sharma MC, Sarkar C, Bhatia R, Singh S, Gulati S, Handa R: Detection of the membrane attack complex as a diagnostic tool in dermatomyositis. Acta Neurol Scand 2011, 123:122-129.

21. Dalakas MC: Sporadic inclusion body myositis: diagnosis, pathogenesis and therapeutic strategies. Nat Clin Pract Neurol 2006, 2:437-447.

22. Ranque B, Berezne A, Le-Guern V, Pagnoux C, Allanore Y, Launay D, Hachulla E, Authier FJ, Gherardi R, Kahan A, Cabane J, Guillevin L, Mouthon L: Myopathies related to systemic sclerosis: a case-control study of associated clinical and immunological features. Scand I Rheumatol 2010, 39:498-505.

23. West SG, Killian PJ, Lawless OJ: Association of myositis and myocarditis in progressive systemic sclerosis. Arthritis Rheum 1981, 24:662-668.

24. Friedrich MG, Sechtem U, Schulz-Menger J, Holmvang G, Alakija P, Cooper LT, White JA, Abdel-Aty H, Gutberlet M, Prasad S, Aletras A, Laissay JP, Paterson I, Filipchuk NG, Kumar A, Pauschinger M, Liu P: Cardiovascular magnetic resonance in myocarditis: a JACC White Paper. J Am Coll Cardiol. 2009, 53:1475-1487.

25. Tyndall AJ, Bannert B, Vonk M, Airo P, Cozzi F, Carreira PE, Bancel DF Allanore $Y$, Müller-Ladner U, Distler O, lannone F, Pellerito R, Pileckyte M, Miniati I, Ananieva L, Gurman AB, Damjanov N, Mueller A, Valentini G, Riemekasten G, Tikly M, Hummers L, Henriques MJ, Caramaschi P, Scheja A, Rozman B, Ton E, Kumánovics G, Coleiro B, Feierl E: Causes and risk factors for death in systemic sclerosis: a study from the EULAR Scleroderma Trials and Research (EUSTAR) database. Ann Rheum Dis 2010, 69:1809-1815

26. Oddis CV, Okano Y, Rudert WA, Trucco M, Duquesnoy RJ, Medsger TA Jr: Serum autoantibody to the nucleolar antigen PM-Scl: Clinical and immunogenetic associations. Arthritis Rheum 1992, 35:1211-1217.

27. Mimori T, Akizuki M, Yamagata H, Inada S, Yoshida S, Homma M: Characterization of a high molecular weight acidic nuclear protein recognized by autoantibodies in sera from patients with polymyositisscleroderma overlap. J Clin Invest 1981, 68:611-620.

28. Aggarwal R, Lucas M, Fertig N, Oddis CV, Medsger TA Jr: Anti-U3 RNP autoantibodies in systemic sclerosis. Arthritis Rheum 2009, 60:1112-1118.

29. Kaji K, Fertig N, Medsger TA Jr, Satoh T, Hoshino K, Hamaguchi Y, Hasewaga M, Lucas M, Schnure A, Ogawa F, Sato S, Takehara K, Fuijmoto M, Kuwana M: Autoantibodies to RuvBL1 and RuvBL2: a novel systemic sclerosisrelated antibody associated with diffuse cutaneous and skeletal muscle involvement. Arthritis Care Res (Hoboken) 2014, 66:575-584.

30. Hoogendijk JE, Amato AA, Lecky BR, Choy EH, Lundberg IE, Rose MR, Vencovsky J, de Visser M, Hughes RA: 119th ENMC international workshop: trial design in adult idiopathic inflammatory myopathies, with the exception of inclusion body myositis, 10-12 October 2003, Naarden, The Netherlands. Neuromuscul Disord 2004, 14:337-345.

31. Varadhachary AS, Weihl CC, Pestronk A: Mitochondrial pathology in immune and inflammatory myopathies. Curr Opin Rheumatol 2010, 22:651-657.

\section{doi:10.1186/ar4562}

Cite this article as: Bhansing et al:: Scleroderma-polymyositis overlap syndrome versus idiopathic polymyositis and systemic sclerosis: a descriptive study on clinical features and myopathology. Arthritis Research \& Therapy 2014 16:R111.

\section{Submit your next manuscript to BioMed Central and take full advantage of:}

- Convenient online submission

- Thorough peer review

- No space constraints or color figure charges

- Immediate publication on acceptance

- Inclusion in PubMed, CAS, Scopus and Google Scholar

- Research which is freely available for redistribution 